\title{
Análisis de la calidad de varios cuerpos de agua superficiales en el GAM y la Península de Osa utilizando el Índice Holandés
}

\author{
Quality analysis of various surface water bodies in the \\ GAM and the osa peninsula \\ using the Dutch Index
}

Guillermo Calvo Brenes'

Jesús Mora Molina ${ }^{2}$

Fecha de recepción: 20 de enero del 2012

Fecha de aprobación:19 de marzo del 2012

Calvo, G; Mora, J. Análisis de la calidad de varios cuerpos de agua superficiales en el GAM y la

Península de Osa utilizando el Índice Holandés. Tecnología en Marcha. Vol. 25, № 5. Pág 37-44. 


\section{Resumen}

A pesar de la cantidad de ríos que tiene Costa Rica, la mayoría muestra algún grado de contaminación. Por lo tanto, resultan de particular interés aquellos que forman parte de la Gran Área Metropolitana, en donde se concentra el 70\% de la población del país.

Si bien la calidad del agua se puede determinar por medio del análisis de distintos indicadores, es ventajosa su simplificación por medio del uso de índices de calidad del agua. Uno de los índices adoptados en Costa Rica es el "Índice holandés de valoración de la calidad del agua" (ICA holandés), el cual se basa en el uso de tres indicadores: la demanda bioquímica de oxígeno, el nitrógeno amoniacal y el oxígeno disuelto, este último expresado como porcentaje de saturación de oxígeno.

En el presente estudio se analizaron estos indicadores en diez ríos localizados en las provincias de San José, Alajuela, Heredia y Cartago, así como en la Península de Osa. Además, para correlacionar el ICA holandés con la densidad poblacional, se muestrearon tres puntos en cada río: la zona alta cercana a la naciente del río, la zona media y la zona baja, ubicada cerca de la desembocadura del río.

Se encontró que los ríos que atraviesan las ciudades altamente densas, las cuales poseen densidades poblacionales superiores a los 8000 habitantes por kilómetro cuadrado (hab $\left./ \mathrm{km}^{2}\right)$, muestran niveles de contaminación en los ríos de "severa" a "muy severa"; por otra parte, lugares con densidades poblacionales entre los 3000-8000 hab/ $\mathrm{km}^{2}$, en general, muestran niveles de contaminación de "moderada" a "severa"; mientras que los poblados con menos de 1000 hab/ $\mathrm{km}^{2}$ causan que la calidad de las aguas presente un nivel de contaminación de "incipiente" a "no contaminada". Estos resultados pueden variar dependiendo del caudal de los ríos, su recorrido total y el uso que se le dé a la tierra.

El análisis de Regresión Lineal Univariable mostró una correlación positiva entre el ICA holandés y la densidad poblacional, y utiliza un nivel de probabilidad del 95\%.

\section{Palabras clave}

Recurso hídrico, contaminación de ríos, Península de Osa, Gran Área Metropolitana, Índice holandés de valoración de la calidad del agua, demanda bioquímica de oxígeno, nitrógeno amoniacal, oxígeno disuelto, densidad poblacional.

\section{Abstract}

Costa Rica is characterized by the amount of rivers, but most of them show high levels of contamination. Special interest is in those of the Gran Area Metropolitana (GAM) since $70 \%$ of the population is located in this area.

Even though there are many water quality indicators, it is advantageous the simplification by the use of water quality index. One of those already adopted in Costa Rica is the Holland Quality Index (Holland ICA). This is based on the use of three parameters: biochemical oxygen demand, ammonium nitrogen and the dissolve oxygen, expressed the last one as saturated oxygen percentage.

This study focused in the analysis of the three parameters in 10 rivers located in the province of San José, Alajuela, Heredia, Cartago, as well as the Península of Osa. For the correlation of the Holland ICA with Population Density, there were sampled 3 places of the same river: one high up place close to the river birth, another in the middle, and the last one, close to the exit.

It was found the rivers going through high density cities with populations over 8000 habitants per square kilometer (hab/ $/ \mathrm{km}^{2}$ ), shows contamination corresponding to "severe" and "highly severe". Places with population between 3000-8000 hab/ $\mathrm{km}^{2}$, in general, shows contamination between "moderate" to "severe". Places with less than $1000 \mathrm{hab} / \mathrm{km}^{2}$ are related to contamination levels of "lightly" to "none". Those results vary depending of rivers flow rate, the total distance alongside the river, as well as the land use.

The Univariate Regression Analysis showed positive correlation between Holland ICA and population density, with a $95 \%$ probability.

\section{Key words}

Hydric resources, rivers contamination, Península de Osa, Gran Area Metropolitana, water quality classification Holland index, biochemical oxygen demand, ammonia nitrogen, dissolved oxygen, population density. 


\section{Introducción}

Costa Rica es un país que posee cantidades importantes de cuerpos de agua, tanto superficiales como subterráneas. Desafortunadamente, los niveles de contaminación de los ríos son altos (Calvo y Mora, 2007a; 2007b; 2007c y 2008). Durante las últimas décadas, Costa Rica ha logrado notables avances en la expansión del acceso a los servicios de abastecimiento de agua, no así la cobertura de los servicios de alcantarillado para la recolección de las aguas residuales domésticas y su subsecuente tratamiento antes de ser vertidas a los ríos (Astorga, 2008). En la actualidad, las aguas residuales domésticas son descargadas a un alcantarillado sanitario o pluvial, o en última instancia, directamente a los cuerpos de agua superficial.

Investigaciones previas demuestran la relación existente entre la densidad poblacional y los niveles de contaminación (Calvo y Mora, 2008); a mayor densidad poblacional, mayor es el grado de contaminación de las aguas superficiales. El crecimiento poblacional del país, desde 1940, ha sido exponencial (INEC, 2008). En el 2009, la población de Costa Rica era de 4509290 habitantes, pero en los últimos años ha mostrado un crecimiento anual a un ritmo alto. Una mayor población implica mayores desechos de residuos sólidos y de aguas residuales, tanto domésticas como industriales (Sevares, 2008).

La determinación de la calidad de un cuerpo de agua usualmente se lleva a cabo por medio del análisis de una cantidad grande de indicadores. Normalmente se expresan en diferentes rangos, distintas unidades y tienen diferente comportamiento en términos de su relación concentración-impacto (Abbasi, 2009). Los indicadores son datos estadísticos o mediciones de una cierta condición, cambio de calidad o cambio de estado de lo que se evalúa. Si varios indicadores se integran de forma que generen un solo valor, se les denomina "índices" (FAO, 200 I).

Uno de los índices adoptados en Costa Rica para medir la calidad de las aguas superficiales es el "Índice holandés de valoración de la calidad para los cuerpos de agua superficiales", según decreto N²5018MEIC publicado en el Diario Oficial La Gaceta $N^{\circ}$ 59 del 25 de marzo de 1996 (MINAE, 2007). El índice se basa en la obtención de un puntaje de acuerdo a varios parámetros analizados: la demanda bioquímica de oxígeno $\left(\mathrm{DBO}_{5}\right)$, el oxígeno disuelto (OD) y el nitrógeno amoniacal $\left(\mathrm{N}-\mathrm{NH}_{4}^{+}\right)$. Este fue el índice utilizado para valorar la calidad del agua en este estudio.

El objetivo de esta investigación fue evaluar la calidad de diez cuerpos de agua en su parte inicial, media y final, pertenecientes a la Cuenca de río Tárcoles, el Reventazón y la Península de Osa. Cada uno de estos puntos presenta características socio-ambientales diferentes en cada uno de sus puntos de muestreo.

\section{Metodología}

Las microcuencas seleccionadas pertenecen a las cuencas del Río Grande de Tárcoles, la cuenca del Reventazón -ambas pertenecientes al GAM, que se caracteriza por albergar el $70 \%$ de la población de todo el país- y la cuenca de los ríos de la Península de Osa.

Los ríos seleccionados fueron el Tigre, el Rincón, el Birrís, el Purires, el Poás, el Agres, el María Aguilar, el Torres, el Segundo y el Cañas. Cada uno de estos ríos se muestreó en la parte alta, cerca de la naciente del río; en su parte media; y en su parte final, cerca de la desembocadura. El monitoreo de los sitios se llevó a cabo por un periodo de doce meses, que inició en noviembre del 2010 y finalizó en octubre del 20 I I. Cada uno de estos puntos fue debidamente georeferenciado.

La valoración de la calidad del agua por el sistema holandés requiere del análisis de tres parámetros: la demanda bioquímica de oxígeno $\left(\mathrm{DBO}_{5}\right)$, el oxígeno disuelto (OD) y el nitrógeno amoniacal $\left(\mathrm{N}_{-} \mathrm{NH}_{4}{ }^{+}\right)$. La metodología, tanto para el análisis como para el muestreo de estos indicadores, fue la establecida en el Standard Methods for the Examination of Water and Wastewater (APHA, 2000). La calidad en términos de estas variables se pondera con una puntuación, indicada en el Reglamento para la clasificación y la evaluación de la calidad de cuerpos de agua superficiales (MINAE, 2007). En la aplicación de este índice, la coloración azul indica un agua no contaminada, el verde corresponde a una contaminación incipiente y el amarillo representa una condición moderada, mientras que el anaranjado es sinónimo de contaminación severa y el rojo de muy severa. 
Cuadro I. Calidad del agua, según el Índice holandés de valoración en las zonas muestreadas

\begin{tabular}{|c|c|c|c|c|c|c|c|c|c|c|c|c|c|}
\hline \multirow{2}{*}{$\begin{array}{l}\text { Punto de } \\
\text { muestreo }\end{array}$} & \multirow{2}{*}{$\begin{array}{l}\text { Densidad } \\
\text { poblacional } \\
\text { (hab/km2) }\end{array}$} & \multicolumn{12}{|c|}{ Mes de muestreo, periodo $2010-2011$} \\
\hline & & Nov & Dec & Ene & Feb & Mar & Abr & May & Jun & Jul & Aug & Set & Oct \\
\hline Ma-I & 0 & 4 & 6 & 5 & 4 & 7 & 5 & 4 & 6 & 9 & 4 & 6 & 4 \\
\hline $\mathrm{Ma}-2$ & 4679 & 5 & 5 & 7 & 10 & 10 & 12 & 8 & 9 & 6 & 5 & 4 & 7 \\
\hline Ma-3 & 10292 & 9 & 10 & 10 & 10 & 12 & 12 & 11 & 11 & 11 & 8 & 7 & 7 \\
\hline To-I & 0 & 4 & 6 & 5 & 4 & 4 & 4 & 5 & 4 & 8 & 4 & 4 & 4 \\
\hline To-2 & 7518 & 8 & 10 & 11 & 13 & 14 & 15 & 13 & 12 & 11 & 10 & 6 & 5 \\
\hline To-3 & 8156 & 8 & 9 & 11 & 13 & 14 & 14 & 12 & 11 & 10 & 12 & 8 & 7 \\
\hline $\mathrm{Se}-1$ & 0 & 5 & 4 & 5 & 4 & 4 & 4 & 4 & 4 & 4 & 6 & 4 & 4 \\
\hline $\mathrm{Se}-2$ & 2911 & 5 & 4 & 3 & 3 & 3 & 4 & 3 & 3 & 5 & 3 & 3 & 3 \\
\hline $\mathrm{Se}-3$ & 3481 & 7 & 6 & 3 & 6 & 4 & 6 & 6 & 5 & 4 & 7 & 6 & 4 \\
\hline Ri-I & 0 & 3 & 4 & 5 & 3 & 1 & 3 & 4 & 5 & 9 & 4 & 4 & 5 \\
\hline Ri-2 & 110 & 3 & 4 & 4 & 3 & 2 & 3 & 4 & 4 & 9 & 3 & 4 & 6 \\
\hline Ri-3 & 2 & 4 & 5 & 4 & 3 & 2 & 4 & 3 & 5 & 6 & 4 & 4 & 5 \\
\hline Ti-I & 4 & 3 & 4 & 5 & 4 & 1 & 4 & 4 & 5 & 4 & 3 & 3 & 6 \\
\hline Ti-2 & 380 & 3 & 5 & 4 & 4 & 1 & 4 & 3 & 5 & 11 & 4 & 4 & 5 \\
\hline Ti-3 & 0 & 3 & 5 & 4 & 3 & 1 & 4 & 7 & 5 & 10 & 5 & 4 & 5 \\
\hline Ca-I & 0 & 4 & 5 & 4 & 3 & 1 & 3 & 4 & 3 & 3 & 3 & 6 & 4 \\
\hline Ca-2 & 3775 & 6 & 8 & 10 & 11 & 3 & 7 & 4 & 7 & 4 & 9 & 4 & 5 \\
\hline Ca-3 & 8982 & 7 & 8 & 9 & 12 & 4 & 11 & 7 & 9 & 9 & 6 & 6 & 6 \\
\hline$A g-1$ & 0 & 4 & 5 & 3 & 3 & 1 & 3 & 3 & 4 & 4 & 3 & 3 & 3 \\
\hline $\mathrm{Ag}-2$ & 1224 & 4 & 4 & 3 & 4 & 2 & 5 & 5 & 7 & 5 & 4 & 4 & 5 \\
\hline $\mathrm{Ag}-3$ & 2145 & 6 & 6 & 7 & 4 & 3 & 6 & 5 & 7 & 9 & 4 & 5 & 4 \\
\hline Po-I & 0 & 4 & 4 & 3 & 4 & 2 & 4 & 3 & 3 & 4 & 3 & 3 & 3 \\
\hline Po-2 & 472 & 4 & 5 & 3 & 3 & 1 & 4 & 4 & 4 & 5 & 3 & 3 & 4 \\
\hline Po-3 & 205 & 5 & 5 & 4 & 3 & 1 & 3 & 3 & 3 & 7 & 3 & 3 & 5 \\
\hline Pu-I & 0 & 4 & 5 & 4 & 4 & 1 & 4 & 4 & 4 & 3 & 4 & 5 & 3 \\
\hline Pu-2 & 257 & 4 & 5 & 4 & 4 & 2 & 3 & 3 & 4 & 5 & 4 & 5 & 5 \\
\hline $\mathrm{Pu}-3$ & 3077 & 8 & 9 & 9 & 9 & 4 & 9 & 12 & 13 & 10 & 10 & 12 & 10 \\
\hline $\mathrm{Bi}-\mathrm{I}$ & 0 & 4 & 4 & 4 & 4 & 1 & 4 & 4 & 5 & 3 & 4 & 3 & 5 \\
\hline $\mathrm{Bi}-2$ & 60 & 5 & 4 & 4 & 3 & 1 & 4 & 3 & 4 & 4 & 4 & 5 & 5 \\
\hline $\mathrm{Bi}-3$ & 306 & 5 & 6 & 6 & 4 & 2 & 4 & 4 & 6 & 7 & 6 & 5 & 5 \\
\hline
\end{tabular}

Notas:

I. Los ríos muestreados fueron el: María Aguilar (Ma), Torres (To), Segundo (Se), Rincón (Ri), Tigre (Ti), Cañas (Ca), Agres (Ag), Poás (Po), Purires (Pu) y Birrís (Bi).

2. Los puntos iniciales se codificaron con un I, los medios con 2 y los finales con 3.

3. Los colores representan los grados de contaminación del agua:
=no contaminada,
=incipiente,
=moderada, =severa,
=muy severa. 


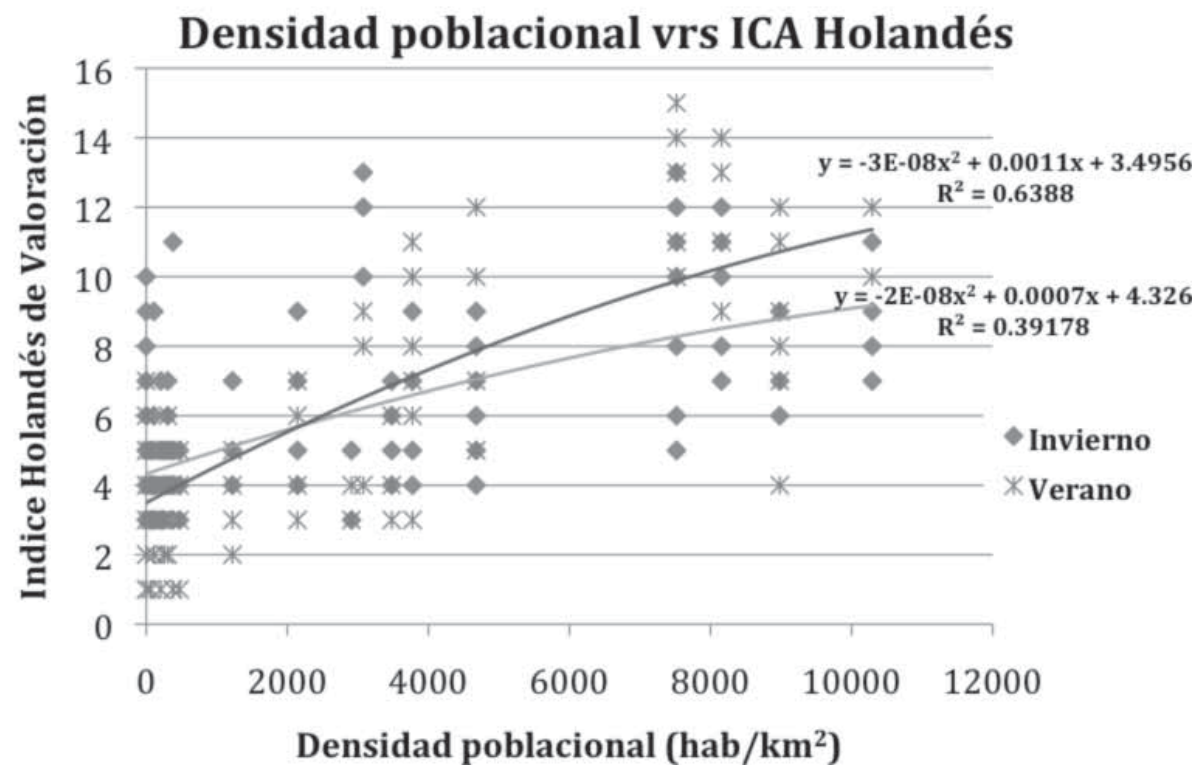

Figura I. Comportamiento del ICA Holandés en relación con la densidad poblacional.

Los datos de densidad poblacional se obtuvieron del IX Censo Nacional efectuado en el 2000, en el cual se reporta los resultados por distritos en cada cantón de cada provincia del país (INEC, 2008). Se procedió a seleccionar aquellos distritos cercanos a los puntos de muestreo y en los que se intuye una influencia en la calidad del agua.

\section{Resultados y discusión}

El cuadro I muestra los resultados encontrados en los treinta puntos muestreados, distribuidos en las diez microcuencas en el periodo que va de noviembre del 2010 a octubre del 201 I, mediante la aplicación del Índice de valoración de la calidad de ríos holandés.

El Instituto Meteorológico Nacional estableció, como época de invierno, los meses que van de mayo hasta mediados de noviembre. Por otra parte, la época de verano corresponde al periodo comprendido entre mediados de noviembre hasta el mes de abril. Cualitativamente, se observa que las microcuencas del Torres y del María Aguilar, que son las que tienen una mayor densidad poblacional, sufrieron un deterioro en la calidad de las aguas en el periodo de verano con respecto al invierno (puntos codificados como 2 y 3 de cada microcuenca). Su clasificación corresponde, en esa época, a contaminación severa o muy severa.

Este efecto puede ser causado por una mayor concentración de los contaminantes en las aguas al disminuir el caudal de los ríos en forma sustancial. Sin embargo, en las zonas con densidad poblacional media-baja, se nota el efecto contrario: en el verano mejora la calidad de las aguas, periodo en que prácticamente cesan las lluvias; tal es el caso de los ríos Tigre, Rincón, Poás y Birrís. Un análisis detallado mostró que este efecto puede ser causado por el contenido de $\mathrm{DBO}$ y la presencia de $\mathrm{N}_{-} \mathrm{NH}_{4}^{+}$, prioritariamente. Aunque el $\mathrm{N}-\mathrm{NH}_{4}{ }^{+}$se encuentra normalmente en aguas superficiales y subterráneas, cambios en los niveles de concentración conllevan sospechas de contaminación antropogénica. En general, la fuente de $\mathrm{N}-\mathrm{NH}_{4}^{+}$se atribuye a la contaminación por fertilizantes o a la contaminación fecal humana y animal.

Pareciera ser que la reducción sustancial del caudal durante la época de verano en zonas altamente densas en población genera la disminución en la calidad del agua por concentración de los contaminantes; mientras que en las otras zonas menos densas, la causa predominante puede ser la disminución en la escorrentía que acarrea el arrastre de $\mathrm{N}-\mathrm{NH}_{4}^{+}$hacia los ríos. 
$42 \mid \begin{aligned} & \text { Tecnología en Marcha, } \\ & \text { Vol. 25, Nº 5, Número Especial } 2012\end{aligned}$

Cuadro 2. Tendencia de los niveles de contaminación durante el verano, según la densidad poblacional

\begin{tabular}{|c|c|c|c|}
\hline \multirow{2}{*}{$\begin{array}{c}\text { Puntaje } \\
\text { según } \\
\text { reglamento }\end{array}$} & Color & Nivel de & Densidad poblacional $\left(\mathrm{Hab} / \mathrm{km}^{2}\right)$ \\
\hline 3 & & contaminación esperado & Época de verano \\
\hline $4-6$ & & Incipiente & $500-3000$ \\
\hline $7-9$ & & Moderada & $3500-6500$ \\
\hline $10-12$ & Severa & $7000-12000$ \\
\hline $13-15$ & Muy severa & $>12500$ \\
\hline
\end{tabular}

En las zonas altamente pobladas, en general, el agua residual doméstica es vertida en los ríos sin tratamiento alguno, de ahí que, en el verano, la concentración de los contaminantes se incremente. El nivel del caudal disminuye notoriamente en los ríos, pero las descargas de aguas residuales domésticas hacia los mismos se mantienen relativamente igual todo el año. En cambio, en zonas rurales donde se localizan el río Rincón, el Tigre, el Poás y el Birrís no ocurren descargas de residuos líquidos directas a los ríos, sino que tal contaminación proviene de los arrastres por escorrentía generada por las lluvias en la época de invierno. Por esta causa, durante el verano, la calidad de estos ríos mejora.

El cuadro I muestra que microcuencas con alta densidad poblacional, como la del Torres y del María Aguilar, poseen contaminaciones catalogadas como "severas" o "muy severas" en varios meses del periodo muestreado. En cambio, microcuencas con baja densidad poblacional, como Rincón y Tigre, presentan una contaminación "incipiente" o "nula". La figura I muestra esta tendencia o correlación al graficarse el Índice de Calidad del Agua (ICA) en función de la densidad poblacional, así como de la estación pluvial. El coeficiente de determinación para el verano es de 0,64, mientras que en el invierno es de 0,39. Significa que durante el verano, el $64 \%$ de la contaminación de los ríos medidos con el Índice holandés es explicado por la densidad poblacional. En cambio, en el invierno, esta contaminación solo es explicada en un 39\% por la densidad poblacional.

El análisis de Regresión Lineal Univariable muestra una correlación positiva entre el ICA Holandés y la densidad poblacional (significación bilateral $\alpha=0,000)$. Como este dato es menor al teórico de 0,05 , se acepta la hipótesis de que existe una correlación positiva entre la densidad poblacional y el grado de contaminación.
Durante el verano, la ecuación que muestra esa correlación es la siguiente:

ICA Holandés $=-3 * 10^{-8 *} \mathrm{DP}^{2}+0,001 \mid$ * DP $+3,4956$

donde DP = densidad poblacional.

Durante el invierno, la ecuación es la siguiente:

$$
\text { ICA Holandés }=-2 * 10-8 * \mathrm{DP}^{2}+0,0007 * \mathrm{DP}+4,326
$$

donde DP = densidad poblacional.

Tanto el río María Aguilar como el Torres son ríos que, en sus nacientes, muestran niveles de contaminación "incipientes" que, conforme atraviesan la ciudad, pasan a niveles de contaminación de "severas" a "muy severas". La densidad poblacional en la ciudad supera los $7000 \mathrm{hab} / \mathrm{km}^{2}$.

El río Rincón y el Tigre de la Península de Osa muestran, en general, una contaminación "incipiente", a pesar de haber una densidad poblacional relativamente baja (menos de $400 \mathrm{hab} / \mathrm{km}^{2}$ ). Pueden atribuirse estos niveles al incremento del uso de la tierra en actividades agrícolas, lo que conlleva a una mayor erosión, especialmente si se considera las fuertes lluvias típicas de la zona, y a la consecuente escorrentía que arrastra material orgánico e inorgánico del suelo hacia los ríos.

Tanto el río Purires como el Cañas muestran contaminaciones de "moderada" a "severa" cuando atraviesan las ciudades de El Tejar en Cartago (3000 hab/ $/ \mathrm{km}^{2}$ ) y Alajuelita en San José (9000 hab/ $\left./ \mathrm{km}^{2}\right)$. El río Agres en su desembocadura muestra una contaminación entre "incipiente" y "moderada"; este río pasa por la ciudad de Escazú con una densidad poblacional de $2000 \mathrm{hab} / \mathrm{km}^{2}$.

El río Segundo muestra niveles de contaminación de "no contaminada" a "incipiente", a pesar de tener densidades poblacionales de 3000-3500 hab/ $\mathrm{km}^{2}$ en los puntos medio y final. Su baja contaminación se 
le atribuye a que el caudal del río es grande, tiene un recorrido total de $30 \mathrm{~km}$ aproximadamente, la mayoría de su trayecto es por zonas poco pobladas $y$, en general, posee una alta cobertura boscosa.

El río Poás y el Birrís presentan una calidad en el agua de "no contaminada" a "incipiente". La densidad poblacional es de 300-400 hab/ $/ \mathrm{km}^{2}$.

El cuadro 2 muestra los posibles niveles de contaminación que tendría el agua superficial de acuerdo con la densidad poblacional. Estos datos corresponden a la época de verano, dado que es la época con el coeficiente de determinación significativamente alto (64\%), mientras que en el invierno este coeficiente es mucho menor. La figura I muestra que existe variabilidad en los valores del ICA holandés para una determinada densidad poblacional. La desviación estándar para los valores del ICA es de 2,5, por lo que es conveniente tener presente que, cuando se predice la asignación del color para una determinada densidad poblacional, puede haber una variación de un nivel de color hacia arriba o hacia abajo del color predicho.

\section{Conclusiones}

La densidad poblacional en las ciudades tiene una incidencia en la calidad de los ríos que las atraviesan. Ciudades con densidades poblacionales superiores a los 8000 hab $/ \mathrm{km}^{2}$ van a presentar niveles de contaminación de "severa"a "muy severa"en aquellos casos que sus aguas no son tratadas adecuadamente antes de ser vertidas directamente a los ríos. Esta condición no solo es desfavorable porque repercute en la calidad de vida en la sociedad, sino que repercute en la contaminación que se traslada a los estuarios y mares, con el consecuente daño en la flora y fauna de dichos hábitats.

Dado el continuo crecimiento poblacional que experimenta nuestro país, las autoridades locales y nacionales deben unir esfuerzos con el fin de mejorar el sistema de alcantarillado para recolectar los desechos líquidos domésticos y darle su posterior tratamiento antes de verterlos en los ríos.

\section{Agradecimientos}

Los autores expresan sus agradecimientos al Consejo Nacional de Rectores (CONARE) por el apoyo financiero, al Instituto Tecnológico de Costa Rica (TEC), en especial a la Vicerrectoría de Investigación y Extensión (VIE), por el apoyo de tiempos y administrativo. También agradecemos al Centro de Investigación en Protección Ambiental (CIPA) y al Laboratorio de Servicios Químicos y Microbiológicos (Ceqiatec) por su gran respaldo al proyecto. Asimismo, expresamos nuestro agradecimiento a la Fundación Neotrópica por el apoyo logístico en la Península de Osa.

Este artículo forma parte del tema de tesis del estudiante de doctorado Guillermo Calvo Brenes dentro del Sistema de Estudios de Postgrado del Programa Doctorado en Ciencias Naturales para el Desarrollo (DOCINADE).

\section{Bibliografía}

1. Abbasi, S.A. (2002). Water quality indices. Published by INCOH Secretariat, National Institute of Hydrology. University of Roorkee, India. Pp. 73.

2. APHA. (2000). Standard methods for the examination of water and wastewater, 20 edición, 2000.

3. Astorga, Y. (2008). Situación del recurso hídrico. Informe preliminar. Decimocuarto informe Estado de la Nación en desarrollo humano sostenible. San José, Costa Rica.

4. Calvo, G \& Mora, J. (2007). Evaluación y clasificación preliminar de la calidad de agua de la cuenca del Río Tárcoles y el Reventazón. Parte II.: Modelo utilizado en la medición de caudales investigados. Tecnología en Marcha, 20(3), Julio-Setiembre, 2007.

5. Calvo, G. \& Mora, J. (2007). Evaluación y clasificación preliminar de la calidad de agua de la cuenca del Río Tárcoles y el Reventazón. Parte III.: Calidad de cuerpos receptores de agua, según el Sistema Holandés de Valoración, Tecnología en Marcha, 20(4), OctubreDiciembre, 2007.

6. Calvo, G. \& Mora, J. (2007). Evaluación y clasificación preliminar de la calidad de agua de la cuenca del río Tárcoles y el Reventazón. Parte l: Análisis de la contaminación de cuatro ríos del área metropolitana. Tecnología en Marcha, 20(2), Abril-Junio 2007. 
7. Calvo, G. \& Mora, J. (2009). Evaluación y clasificación preliminar de la calidad de agua de la cuenca del Río Tárcoles y el Reventazón. Parte IV. Análisis estadístico entre variables. Tecnología en Marcha, 22(I), EneroMarzo 2009.

8. FAO. (200 I). Indicadores de la calidad de la tierra y su uso para la agricultura sostenible y el desarrollo rural. Boletín de tierras y aguas de la FAO. http:// uned.blackboard.com/webct/urw/lc5 I I 600 I.tp0/ cobaltMainFrame.dowebct (I5/06/2009).
9. INEC. (2008). Estimaciones y proyecciones de población por sexo y edad (cifras actualizadas) 1950-2050. http:// www.inec.go.cr/Web/Home/GeneradorPagina.aspx. $(|| /|| /||)$.

10.MINAE. (2007). Reglamento para la clasificación y la evaluación de la calidad de cuerpos de agua superficiales. Decreto 33903 del 17 de Setiembre del 2007. Diario Oficial La Gaceta. Número 129.

11. Sevares, J. (2008). El imperio de las Finanzas sobre las economías, las empresas y los ciudadanos. Grupo Editorial Norma. Pp 98- 447. 